\title{
INVOLUTIVE FORMULATION AND SIMULATION FOR ELECTRONEUTRAL MICROFLUIDS
}

\author{
Bijan Mohammadi ${ }^{1}$ And Jukka TuOmela ${ }^{2}$
}

\begin{abstract}
We study a microfluidic flow model where the movement of several charged species is coupled with electric field and the motion of ambient fluid. The main numerical difficulty in this model is the net charge neutrality assumption which makes the system essentially overdetermined. Hence we propose to use the involutive and the associated augmented form of the system in numerical computations. Numerical experiments on electrophoresis and stacking show that the completed system significantly improves electroneutrality constraint conservation and recovers analytical results while a direct implementation of the initial model fails.
\end{abstract}

Mathematics Subject Classification. 35K55, 58J10, 65M60, 76W05.

Received September 17, 2009.

Published online April 15, 2011.

\section{INTRODUCTION}

Over the past 15 years, integrated electrokinetic microsystems have been developed with a variety of functionalities including sample pretreatment, mixing, and separation. Electric fields are used either to generate bulk fluid motion (electroosmosis) or to separate charged species (electrophoresis). In this article we study a widely used mathematical model for electrophoresis $[2,3,7,10,18,22]$.

Mathematically this leads to a complicated system of nonlinear PDEs. The system is naturally composed of 2 blocks. The first is the Stokes system which governs the motion of the ambient fluid where the charged species of interest are. The second block is a nonlinear convection diffusion system for charged species and elliptic equation for electric potential. The real challenge comes from the physically reasonable charge neutrality assumption: charge distributions for different species add up to zero. This constraint makes the system essentially overdetermined, and leads immediately to problems with standard numerical methods which are designed to deal only with square systems (as many equations as unknowns).

Our method, already used in [12,13], uses the involutive and its associated augmented form of the system in numerical computations. The augmented form can be constructed once the involutive system and its compatibility operator are known. Typically this leads us back to the class of square systems where again standard methods are applicable. All relevant constraints of the problem are now explicitly included in the augmented system, hence the numerical errors related to constraints can effectively be controlled. The computations thus

\footnotetext{
Keywords and phrases. Microfluids, electrophoresis, stacking, overdetermined PDE systems, involution.

${ }^{1}$ Institut de Mathématiques et Modélisation de Montpellier, Université Montpellier II, France. bijan.mohammadi@univ-montp2 . fr

2 Department of Physics and Mathematics, University of Eastern Finland, Finland. jukka.tuomela@uef.fi
} 
become more stable because one does not need to worry about possible instabilities due to nonrespect of constraints. Also the results will be more reliable, and they are more likely to reproduce essential qualitative properties of the solution than the standard methods. An example of this is presented below. Note that our approach is not restricted to microfluidic systems, but on the contrary can be applied in a wide variety of situations.

The content of the article is as follows. In Section 2 we briefly recall what is meant by involutive, completed and augmented systems. In Section 3 we present the physical model and derive the corresponding completed and augmented systems. Then in Section 4 we analyse the symbol of the augmented model. This analysis suggests a definition of a new class of systems which we call systems of elliptic-parabolic type. As far as we know this definition has not appeared before in the literature. In Section 5 we present our numerical results. Our method gives clearly better results than the standard approach. Finally in Section 6 we conclude with some perspectives for future work.

\section{Involutive, COMPLETED AND AUGMENTED SYSTEMS}

\subsection{Involutive systems}

The important concept of involutivity is unfortunately quite difficult to define precisely. However, for our purposes it is sufficient to explain the idea in concrete terms and indicate how to work with this notion constructively. For more details we refer to $[5,8,9,12,13,17,19,21]$.

Let us consider the system $\nabla \times y+y=0$. Taking the divergence we see that if $y$ is a solution, then it must also satisfy $\nabla \cdot y=0$. This new equation is called a differential consequence or integrability condition of the initial system. Hence we have two systems:

$$
\mathcal{S}: \nabla \times y+y=0, \quad \mathcal{S}^{\prime}:\left\{\begin{array}{l}
\nabla \times y+y=0 \\
\nabla \cdot y=0
\end{array}\right.
$$

We say that $\mathcal{S}^{\prime}$ is the involutive form of $\mathcal{S}$ because no more new first order differential consequences can be found. So informally we may say that a system is involutive, if it contains all its differential consequences (up to given order).

There are many tricky issues involved when one actually tries to compute the involutive form, but the important point is that these constructions can be in fact carried out. Hence the approach we are proposing here is potentially useful for solving quite general systems of PDE.

It turns out that for the purposes of numerical computation it is sometimes convenient to use not the "full" involutive form of the system, but to add just some of the integrability conditions to the original system. Hence we will use the term completed system to indicate that we may not use the full involutive system.

But since completed systems usually have more equations than unknowns while the numerical methods for PDEs are designed for square systems, it is not obvious how to generalize for example the finite element method to this more general case. We outline below one possible approach.

\subsection{Augmented systems}

Let us consider our problem in a general form

$$
A_{0} y=f
$$

and let us suppose that $A_{0}$ is already in completed form. Now since $A_{0}$ is in general overdetermined, there are typically no solutions for arbitrary $f$; hence there are some compatibility conditions for $f$. These conditions are given by a compatibility operator $A_{1}$ such that $A_{1} A_{0}=0$ and (2.2) has a solution only if $A_{1} f=0$. 
Let us now introduce some function spaces $V_{i}$ such that $A_{i}: V_{i} \rightarrow V_{i+1}$. Let us suppose that $A_{0}$ is injective, $A_{1}$ is surjective, and that image $\left(A_{0}\right)=\operatorname{ker}\left(A_{1}\right)$; in other words the following complex is exact:

$$
0 \rightarrow V_{0} \stackrel{A_{0}}{\longrightarrow} V_{1} \stackrel{A_{1}}{\longrightarrow} V_{2} \rightarrow 0 .
$$

This suggests that we can decompose $V_{1}$ as follows:

$$
\operatorname{image}\left(A_{0}\right) \oplus \operatorname{image}\left(A_{1}^{T}\right) \simeq V_{1},
$$

where $A_{1}^{T}$ is the formal transpose of $A_{1}$. Of course to be able to write equality instead of $\simeq$ we should specify carefully the relevant vector spaces. However, proceeding formally, this decomposition suggests that it is indeed possible to find some functional framework such that the combined operator $\left(A_{0}, A_{1}^{T}\right)$ would be bijective or at least Fredholm.

So instead of trying to solve the original system (2.2) in some least square sense, we introduce an auxiliary variable $\tilde{y}$ and solve

$$
A_{0} y+A_{1}^{T} \tilde{y}=f .
$$

We call this system the augmented system. This formulation is reasonable because the augmented system is square, hence standard software is readily available. Also all the relevant information about the original system is contained in the completed operator $A_{0}$ which means that the results will be reliable. The drawback is that we have introduced an extra variable $\tilde{y}$ which increases the computational cost. However, we can use $\tilde{y}$ in error control as explained in [12].

\section{Governing Equations of IONIC MiCROFluids}

\subsection{Physical background}

Electrokinetic systems have been developed to perform a variety of functions including chemical separations, pre-concentration, and mixing [3,22]. Examples of separation assays include on-chip capillary zone electrophoresis and isoelectric focusing. Preconcentration methods include field amplified sample stacking and isotachophoretic preconcentration. These applications involve the convective-diffusion-electromigration of multiple ionic and neutral species.

Below we will discuss a fairly general convective diffusion system of equations applicable to electrokinetic microfluidics. We assume uniform and constant electrical permittivity and low Reynolds number flows. We also assume species are dilute enough to apply the Nernst-Planck equations and the dilute approximation for Fick's law [18].

Our formulation is applicable in thin electrical double layers, and accounts for net charge accumulation in the bulk resulting from a coupling between electric fields and conductivity gradients. The principal computational difficulty in this model is due to the net electroneutrality assumption. This assumption is valid on the length scales relevant to the phenomena under study $[7,10,18]$.

\subsection{Ionic flows modelling}

Let us consider the case where we have $m$ different charged species in some ambient fluid. Let $\rho^{e}=$ $F \sum_{i} z_{i} C^{i}=\sum_{i} z_{i} c^{i}$ be the net charge density where $z_{i} \in \mathbb{Z}$ is the valence number of species $i, C^{i}$ is the molar concentration and $F$ is the Faraday's constant. The charge induces an electric field which is supposed to come from the potential $\phi$. Hence we get our first equation

$$
-\varepsilon \Delta \phi-\rho^{e}=0,
$$

where $\varepsilon$ is the permittivity of the ambient fluid. The motion of ambient fluid is governed by Navier-Stokes equations, but because in typical applications the Reynolds number is very low we can as well use the Stokes 
system. We will further suppose that the ambient fluid is incompressible. Hence the Stokes system in the presence of electric field can be written as

$$
\begin{aligned}
\rho u_{t}-\mu \Delta u+\nabla p-\rho^{e} \nabla \phi & =0, \\
\nabla \cdot u & =0 .
\end{aligned}
$$

Here $\rho$ is the density of the fluid and $\mu$ is the dynamic viscosity. Now the movement of different species are governed by equations $c_{t}^{i}+\nabla \cdot J^{i}=0$ where the current density $J^{i}$ is given by

$$
\mathrm{J}^{i}=-\nu_{i} z_{i} c^{i} \nabla \phi-d_{i} \nabla c^{i}+c^{i} u,
$$

where $\nu_{i}$ is the mobility times the Faraday's constant and $d_{i}$ the diffusivity of species $i$. We have now introduced all necessary variables and parameters. Taking into account that $\nabla \cdot u=0$ one has:

$$
\nabla \cdot \mathrm{J}^{i}=-d_{i} \Delta c^{i}-\nu_{i} z_{i} \nabla \cdot\left(c^{i} \nabla \phi\right)+u \cdot \nabla c^{i} .
$$

This gives the system

$$
\begin{aligned}
\rho u_{t}-\mu \Delta u+\nabla p-\rho^{e} \nabla \phi & =0, \\
\nabla \cdot u & =0, \\
c_{t}^{i}-d_{i} \Delta c^{i}-\nu_{i} z_{i} \nabla \cdot\left(c^{i} \nabla \phi\right)+u \cdot \nabla c^{i} & =0, \quad i=1, \ldots, m \\
-\varepsilon \Delta \phi-\rho^{e} & =0, \\
\rho^{e}-\sum_{i} z_{i} c^{i} & =0 .
\end{aligned}
$$

\subsection{The net neutrality situation}

In ionic flows, one important situation is net neutrality where $\rho^{e}=0[7,18]$. This equilibrium situation is usually spatially dominant. This introduces an algebraic constraint and our aim through this paper is to show how to numerically verify this constraint without removing one of the variables as shown below.

With the net charge set to zero and taking the divergence of the first equation and simplifying we obtain

$$
\begin{aligned}
\rho u_{t}-\mu \Delta u+\nabla p & =0, \\
-\Delta p & =0, \\
\nabla \cdot u & =0, \\
c_{t}^{i}-d_{i} \Delta c^{i}-\nu_{i} z_{i} \nabla \cdot\left(c^{i} \nabla \phi\right)+u \cdot \nabla c^{i} & =0, \quad i=1, \ldots, m \\
-\Delta \phi & =0, \\
-\sum_{i} z_{i} c^{i} & =0 .
\end{aligned}
$$

Note that the system is naturally composed of 2 blocks: the Stokes system for variables $(u, p)$ and a diffusion like system for variables $(c, \phi)$.

The main difficulty in solving (3.1) with standard methods is that the electroneutrality constraint (the last equation) is not respected. Of course one of the species could be deduced from the constraint:

$$
c^{m}=-\frac{1}{z_{m}} \sum_{i=1}^{m-1} z_{i} c^{i} .
$$


However, this is a real limitation because in this case the physical characteristics of one species are not taken into account during integration. Hence it is definitely interesting to include the electroneutrality constraint explicitly in the numerical model.

To complete the model (3.1) we should now impose the relevant boundary conditions. However, now an essential difficulty appears concerning the potential $\phi$. On one hand from physical point of view it is clear that values of $\phi$ on the boundary depend on other variables of the problem. However, in order to get physically reasonable boundary conditions one should model the quite complicated interactions of variables in a thin boundary layer. On the other hand in the intended application the precise behavior of the solution in the boundary layer is not very important and hence one would like to use some simple boundary conditions and model the interactions of potential with other variables in another way. Hence we drop the equation $\Delta \phi=0$ in (3.1) and proceed with

$$
\begin{aligned}
\rho u_{t}-\mu \Delta u+\nabla p & =0, \\
-\Delta p & =0, \\
\nabla \cdot u & =0, \\
c_{t}^{i}-d_{i} \Delta c^{i}-\nu_{i} z_{i} \nabla \cdot\left(c^{i} \nabla \phi\right)+u \cdot \nabla c^{i} & =0, \quad i=1, \ldots, m \\
-\sum_{i} z_{i} c^{i} & =0 .
\end{aligned}
$$

Above we have only briefly indicated some basic issues in the derivation of the mathematical model (3.3). This model is firmly established and widely used in the simulation of electrophoresis. The reader who wishes to learn more about the physical details and relevant assumptions behind this model can find a thorough treatment in $[7,18]$.

\subsection{Completed and augmented systems}

It is convenient to introduce the following quantity:

$$
\psi=\sum_{i=1}^{m} z_{i}^{2} \nu_{i} c^{i}
$$

Note that $\psi$ remains strictly positive because there is necessarily at least one species present in the flow.

Let us consider the system (3.3). There seems to be too few equations: there is apparently no "natural" $2^{\text {nd }}$ order equation for $\phi$. However, we can get an independent equation for $\phi$ using the "constraint" $\sum_{i} z_{i} c^{i}=0$. Differentiating this we obviously have

$$
\sum_{i} z_{i} \Delta c^{i}=0
$$

We now multiply the equations for $c^{i}$ by $z_{i}$, then operate with $\partial_{t}-a \Delta$ to the last equation and add. This gives

$$
\begin{aligned}
\rho u_{t}-\mu \Delta u+\nabla p & =0, \\
-\Delta p & =0, \\
-\nabla \cdot u & =0, \\
c_{t}^{i}-d_{i} \Delta c^{i}-\nu_{i} z_{i} \nabla \cdot\left(c^{i} \nabla \phi\right)+u \cdot \nabla c^{i} & =0, \quad i=1, \ldots, m \\
-\nabla \cdot(\psi \nabla \phi)-\sum_{i} z_{i}\left(d_{i}-a\right) \Delta c^{i} & =0, \\
-\sum_{i} z_{i} c^{i} & =0 .
\end{aligned}
$$


Our next task is to construct the augmented system corresponding to (3.5), and in order to do that we need to find the compatibility operator. Let us denote $y=\left(u^{1}, u^{2}, u^{3}, p, c^{1}, \ldots, c^{m}, \phi\right)$ and write the system (3.5) as $A_{0} y=0$. Now the Stokes system is a subsystem of (3.5), hence one compatibility condition comes from that. The second one is simply given by our construction of the equation for $\phi$. So if we define

$$
A_{1}=\left(\begin{array}{cccccccc}
\nabla \cdot 1 & \rho \partial_{t}-\mu \Delta & 0 & \ldots & 0 & 0 & 0 \\
0 & 0 & 0 & z_{1} & \ldots & z_{m} & -1 & \partial_{t}-a \Delta
\end{array}\right)
$$

then it is easy to check that $A_{1} A_{0}=0$. Now introducing $\tilde{y}=\left(\tilde{y}^{1}, \tilde{y}^{2}\right)$ the augmented system $A_{0} y+A_{1}^{T} \tilde{y}=0$ can be written as

$$
\begin{aligned}
\rho u_{t}-\mu \Delta u+\nabla p-\nabla \tilde{y}^{1} & =0, \\
-\Delta p+\tilde{y}^{1} & =0, \\
\rho \tilde{y}_{t}^{1}-\nabla \cdot u-\mu \Delta \tilde{y}^{1} & =0, \\
c_{t}^{i}-d_{i} \Delta c^{i}-\nu_{i} z_{i} \nabla \cdot\left(c^{i} \nabla \phi\right)+u \cdot \nabla c^{i}+z_{i} \tilde{y}^{2} & =0, \quad i=1, \ldots, m \\
-\nabla \cdot(\psi \nabla \phi)-\sum_{i} z_{i}\left(d_{i}-a\right) \Delta c^{i}-\tilde{y}^{2} & =0, \\
\tilde{y}_{t}^{2}-a \Delta \tilde{y}^{2}-\sum_{i} z_{i} c^{i} & =0 .
\end{aligned}
$$

Note that the system (3.6) is nicely decoupled in two blocks so that the auxiliary variables do not interact.

It is important to note that the systems (3.3) and (3.5) are formally equivalent; by this we mean that every (sufficiently regular) solution of (3.3) is also a solution of (3.5) and vice versa. On the other hand the system (3.6) is not formally equivalent to (3.3) and (3.5). The system (3.6) is important because with it we can compute in a stable way good approximations to the overdetermined problem (3.5).

Note also that the equation

$$
-\nabla \cdot(\psi \nabla \phi)-\sum_{i} z_{i}\left(d_{i}-a\right) \Delta c^{i}-\tilde{y}^{2}=0
$$

in (3.6) is nonlinear since $\psi$ depends on $c^{i}$ by (3.4). This (and the corresponding equation in (3.5)) was only "hidden" in the system (3.3). This is the interest of completing the systems in general: the analysis reveals explicitly the properties of the systems. Once these formal properties of the system are known, it is then much easier to design numerical methods which preserve these properties.

\subsection{Some comparisons between different formulations}

The systems (3.3) and (3.6) are complicated nonlinear systems and we are not aware that there are any rigorous results concerning the existence and uniqueness of their solutions. However, we can still argue that there are good reasons to believe that the augmented system (3.6) is "better" than the system (3.3). This can be seen by analysing the properties of the symbol of different models.

The general idea here is that completing the system will also "improve" the symbol and hence the whole operator in the following sense. Douglis and Nirenberg [1,4] (resp. Solonnikov [6,20]) generalized the notion of ellipticity (resp. parabolicity) by introducing certain weights for the variables and equations. However, we proved $[8,9]$ that such systems always become elliptic or parabolic in the standard sense when completed. Moreover, there are systems which are not elliptic or parabolic in the generalised sense initially but which become 
elliptic or parabolic when completed. For example the operator $\mathcal{S}$ in (2.1) is not elliptic even in generalised sense but the completed system $\mathcal{S}^{\prime}$ is elliptic.

In the present case the system is neither elliptic nor parabolic, but intuitively we could say that $(u, c, \tilde{y})$ are parabolic variables and $(p, \phi)$ are elliptic variables. Let us note that some chemotaxis models also lead to problems of this type [15]. The main point here is that when we complete the system with the equation

$$
-\nabla \cdot(\psi \nabla \phi)-\sum_{i} z_{i}\left(d_{i}-a\right) \Delta c^{i}=0
$$

the system (3.5) is elliptic in a stronger sense with respect to $\phi$ than the system (3.3). Also (3.6) is strongly elliptic with respect to $\phi$. Strictly speaking this analysis applies only to the linearised systems. However, since the numerical solution of nonlinear systems involves solving iteratively linearised problems, in typical situations the well-posedness of the linearised problem is a necessary condition for a successful solution of the nonlinear problem.

\section{IMPLEMENTATION AND EXAMPLES}

\subsection{Physical setting}

The derivation of completed and augmented systems presented above is valid in any dimension. Physically the problem is obviously strictly speaking 3 dimensional. In a typical application one could consider a horizontal channel of $100 \mu \mathrm{m}$ wide, $100 \mu \mathrm{m}$ deep and several centimeters long. However, the boundary layers along channel walls are only a few nanometers thick which implies that a 2 dimensional model is relevant and even a 1 dimensional model can be used [2]. Indeed when one is mainly interested in stacking (see below) already 1 dimensional model gives sufficiently accurate results. However, if one wants to simulate how the propagating front of some concentration is distorted for example in curved channels then one must use a 2 dimensional model.

We did some computations both with 2 and 1 dimensional models. The channel width was chosen as $100 \mu \mathrm{m}$ and the length as $1 \mathrm{~mm}$. The computational cost of 2 dimensional case was about 100 times bigger than in 1 dimensional case. Such a dramatic difference is due to the fact that the resolution of the flow of the ambient fluid becomes trivial in 1 dimensional case. Hence we did only few test runs in 2 dimensional case and most of the computations were done with 1 dimensional model.

Because we have already analysed the augmented Stokes system in our previous papers $[12,13]$ we will only discuss below how our formulation improves the quality of the solutions of concentrations of species $c^{i}$. This is also justified by the fact that in this model the main interest and the main computational difficulty is related to concentrations while the motion of the ambient fluid is relatively unimportant and in any case considerably easier to compute.

\subsection{Stacking}

The aim of stacking is to amplify the local concentrations of species to make them observable by existing devices. We consider the case of 3 ionic species. The third species is the sample species of interest whose molar concentration is low and one would like to increase it through stacking. Typically the sample species is three or four orders of magnitude smaller than other species and consequently one needs to stack the sample species by the factor of about 1000 to make it observable. Representative experimental conditions are described in $[2,10,14]$. 
To model a single interface of conductivities, we choose a simple error function profile for the initial concentration. We consider the following initial conditions for the species that reproduces well the experimental conditions:

$$
\begin{aligned}
c_{\text {init }}^{1}(x) & =\frac{1}{2} b_{1}(\gamma+1-(\gamma-1) \operatorname{erf}(\alpha x)), \\
c_{\text {init }}^{3}(x) & =b_{3}(1+\operatorname{erf}(\alpha x)), \\
z_{2} c_{\text {init }}^{2}(x) & =-z_{1} c_{\text {init }}^{1}(x)-z_{3} c_{\text {init }}^{3}(x),
\end{aligned}
$$

where $b_{1}=100, b_{3}=0.1, \alpha>0$ and $\gamma>0$. Note that $\alpha$ controls the sharpness in the initial plug and $\gamma$ indicates the initial concentration ratio between high and low conductivity regions in the sense that

$$
\lim _{x \rightarrow-\infty} c_{\text {init }}^{1}(x) / b_{1}=\gamma, \quad \lim _{x \rightarrow \infty} c_{\text {init }}^{1}(x) / b_{1}=1 .
$$

In fact a more important property of $\gamma$, both experimentally and numerically, is that it also indicates the final stacking ratio of $c^{3}$. This can be seen as follows.

Let us consider the 1 dimensional model. Neglecting diffusion and the motion of the ambient fluid, the species conservation equation for different species becomes

$$
c_{t}^{i}-\frac{\partial}{\partial x}\left(z_{i} \nu_{i} c^{i} \phi_{x}\right)=0
$$

A constant current density $\mathrm{j}$ is applied in the axial direction defined by the difference of electric potential at the ends of the channel:

$$
\mathrm{j}=\frac{V_{\text {left }}-V_{\text {right }}}{L} \sigma_{\text {total }}
$$

where $L$ is the length of the channel and

$$
\sigma_{\text {total }}=\int_{0}^{L} \sigma(x, t) \mathrm{d} x, \quad \sigma(x, t)=\sum_{i} z_{i}^{2} \nu_{i} c^{i} .
$$

Let us further suppose that $\sigma_{\text {total }}$ is independent of time. The electric field is then locally defined as:

$$
E=\phi_{x}=\frac{j}{\sigma}
$$

Now in our setting of 3 species the concentration of sample species $c^{3}$ increases as it migrates from a region of high conductivity to a region of lower drift velocity. Hence the stacked sample keeps increasing with time and the concentration progressively approaches a maximum steady value. Now in the steady state the net flux of the species $c^{3}$ at the left and right edges balance:

$$
\left.\left(z_{3} \nu_{3} c^{3} E\right)\right|_{\text {left }}=\left.\left(z_{3} \nu_{3} c^{3} E\right)\right|_{\text {right }} .
$$

Then considering the initial conditions (4.1) we have

$$
\sigma_{\text {left }}=\sum_{i=1}^{3} z_{i}^{2} \nu_{i} c_{\text {left }}^{i} \approx z_{1}^{2} \nu_{1} c_{\text {left }}^{1}+z_{2}^{2} \nu_{2} c_{\text {left }}^{2} \approx\left(z_{1}^{2} \nu_{1}-z_{1} z_{2} \nu_{2}\right) b_{1} \gamma
$$

As $\mathrm{j}$ is constant this implies

$$
\frac{c_{\text {left }}^{3}}{c_{\text {right }}^{3}}=\frac{E_{\text {right }}}{E_{\text {left }}}=\frac{\sigma_{\text {left }}}{\sigma_{\text {right }}} \approx \gamma
$$


When diffusion and motion of the ambient fluid are present the above arguments can still be used to provide an upper bound for the stacking capacity. Indeed, both diffusion and the convective motion tend to distort the front where stacking happens and hence one expects a lower final stacking ratio in this case.

\subsection{Boundary conditions}

The boundary condition for the Stokes system is classical except along solid walls where, to avoid treating the nanometric double-layer region, one assumes the following slip boundary condition $[7,18]$ :

$$
u=-\zeta \nabla \phi
$$

Here $\zeta$ is a positive constant which depends on the material with which channels are built and also the fluid permittivity and dynamic viscosity. The flow is therefore parallel to the wall and to the electric field. For the electric potential we impose a Dirichlet boundary condition at the inlet and the outlet where a difference of potential is applied and homogeneous Neumann boundary condition is assumed along the wall. Together with the boundary condition for $u$ along the wall, the former condition enforces the non-penetration boundary condition for the velocity.

The boundary condition for the concentration is Dirichlet at the inlet boundary, homogeneous Neumann along the walls and at the outlet. Initial and boundary conditions for the auxiliary variables $\tilde{y}$ are identically zero as these are compatible with the requirement that the solution of the initial and completed systems should match.

\subsection{Algorithm}

Whether solving the initial (3.3) or augmented system (3.6) the system is basically composed of 2 blocks: the Stokes problem for the velocity and pressure and some kind of convection diffusion system for the concentrations and the electric field. To make the implementation of this coupled system efficient we need to be able to use black-box solvers or existing pieces of validated software. This is only possible if we can solve coupled systems cyclically. At the same time we would like to make sure that the order in which the variables are solved does not influence the overall solution. To satisfy these requirements we use the following fixed point iterations where $\tau$ indicates a fictitious time. This transient fixed point algorithm, where at each real time interation, one iterates in a fictiotious time until convergence is convenient for solving coupled systems cyclically.

Let $y$ be the vector of variables $y=\left(u, p, \phi, c^{1}, . ., c^{m}, \tilde{y}^{1}, \tilde{y}^{2}\right)$ as before and let us rewrite our models as $(\mathcal{M}=\operatorname{diag}(1,0,0,1, . ., 1))$ :

$$
F\left(\mathcal{M} y_{t}, y\right)=0, \quad y(0)=y_{0} .
$$

Then we can write the global algorithm in the following form.

$$
\begin{aligned}
& \begin{array}{l}
y^{0}:=y_{0} \\
\text { FOR } q=0,1, \ldots \\
\bar{y}^{0}:=y^{q} ; k:=0 \\
\text { REPEAT } \\
\quad k:=k+1 \\
\quad \frac{\bar{y}^{k}-\bar{y}^{k-1}}{\Delta \tau}+\tilde{F}\left(\mathcal{M} \frac{\bar{y}^{k}-y^{q}}{\Delta t}, \bar{y}^{k}, \bar{y}^{k-1}\right)=0 \\
\quad \text { UNTIL }\left\|\bar{y}^{k}-\bar{y}^{k-1}\right\| \leq \text { tol } \\
y^{q+1}:=\bar{y}^{k}
\end{array}
\end{aligned}
$$

ENDFOR 
Because we look for the steady solution when iterating in $\tau$, the order in which the equations are solved cyclically has no importance. One should choose the ordering which advantages implicitness. For instance, in the case of the augmented system (3.6) $F\left(\mathcal{M} \frac{\bar{y}^{k}-y^{q}}{\Delta t}, \bar{y}^{k}, \bar{y}^{k-1}\right)=0$ encapsulates the following relations:

$$
\begin{aligned}
& \rho \frac{\overline{\tilde{y}}^{k}-\left(\tilde{y}^{1}\right)^{q}}{\Delta t}-\nabla \cdot \bar{u}^{k-1}-\mu \Delta{\overline{\tilde{y}^{1}}}^{k}=0, \\
& -\Delta \bar{p}^{k}+{\overline{\tilde{y}^{1}}}^{k}=0 \\
& \rho \frac{\bar{u}^{k}-u^{q}}{\Delta t}-\mu \Delta \bar{u}^{k}+\nabla \bar{p}^{k}-\nabla{\overline{\tilde{y}^{1}}}^{k}=0, \\
& \text { with in particular } \bar{u}^{k}=-\zeta \nabla \bar{\phi}^{k-1} \text { along solid walls, } \\
& \frac{{\overline{c^{i}}}^{k}-\left(c^{i}\right)^{q}}{\Delta t}-d_{i} \Delta{\overline{c^{i}}}^{k}-\nu_{i} z_{i} \nabla \cdot\left({\overline{c^{i}}}^{k} \nabla \bar{\phi}^{k-1}\right)+\bar{u}^{k} \cdot \nabla{\overline{c^{i}}}^{k}+z_{i}{\overline{\tilde{y}^{2}}}^{k-1}=0 \text {, } \\
& i=1, \ldots, m \\
& -\nabla \cdot\left(\bar{\psi}^{k} \nabla \bar{\phi}^{k}\right)-\sum_{i} z_{i}\left(d_{i}-a\right) \Delta{\overline{c^{i}}}^{k}-\overline{\tilde{y}}^{k-1}=0 \\
& \text { with } \bar{\psi}^{k}=\sum_{i} z_{i}^{2} \nu_{i} \bar{c}^{k} \\
& \frac{{\overline{\tilde{y}^{2}}}^{k}-\left(\tilde{y}^{2}\right)^{q}}{\Delta t}-a \Delta{\overline{\tilde{y}^{2}}}^{k}-\sum_{i} z_{i}{\overline{c^{i}}}^{k}=0 .
\end{aligned}
$$

Hence, the map $F$ contains implicitly several solvers. It is an upgrade of the code presented in [2] where electroneutrality constraint is artificially enforced removing one of the species. Because the geometry is simple we used quadrilateral meshes. First we have Stokes or augmented Stokes solver for $u$ and $p$. The classical Stokes solver needs no comment and the augmented Stokes system is described in [12,13]. Note that we have used equal order discretizations for $u$ and $p$ in the augmented system because in our approach we do not need to worry about infsup-condition. Then there are calls to a transport-diffusion-reaction solver for each species $c^{i}$. Once $c^{i}$ have been computed we can form $\psi=\sum_{i} z_{i}^{2} \nu_{i} c^{i}$ and then use a Poisson solver for $\phi$. In the case of the augmented system we need in addition two calls to a heat equation solver with source terms for the auxiliary variables.

The standard numerical viscosity techniques are used with the advection both in the standard case and the augmented case because our approach does not remove the need for such stabilizations. The prolems in the numerical treatment of advection have different origins and are not related to the treatment of differential (zero divergence) or algebraic (electroneutrality) constraints which are handled in a better way in the augmented system.

This algorithm eventually provides fully implicit coupling. Indeed, after reaching steady intermediate states in $\tau$ one takes $y^{q+1}=\bar{y}^{k}=\bar{y}^{k-1}$ which gives $\tilde{F}\left(\mathcal{M} \frac{y^{q+1}-y^{q}}{\Delta t}, y^{q+1}, y^{q+1}\right)=F\left(\mathcal{M} \frac{y^{q+1}-y^{q}}{\Delta t}, y^{q+1}\right)=0$.

If greater accuracy in time is desired the above scheme can be readily adapted to the case of higher order difference schemes. Also faster convergence in the inner loop can be achieved using standard convergence acceleration techniques.

\subsection{Test cases}

We have used the following parameters in our computations, given in SI units. Recall that mobility in our case is the usual mobility multiplied by Faraday's constant.
mobility
diffusivity
$\nu_{1}=5 \times 10^{-8}$
$\nu_{2}=3 \times 10^{-7}$
$d_{2}=3 \times 10^{-10}$
$\nu_{3}=3 \times 10^{-8}$
valence number
$d_{1}=2 \times 10^{-10}$
$z_{2}=-1$
$d_{3}=2 \times 10^{-10}$
$z_{3}=-2$. 

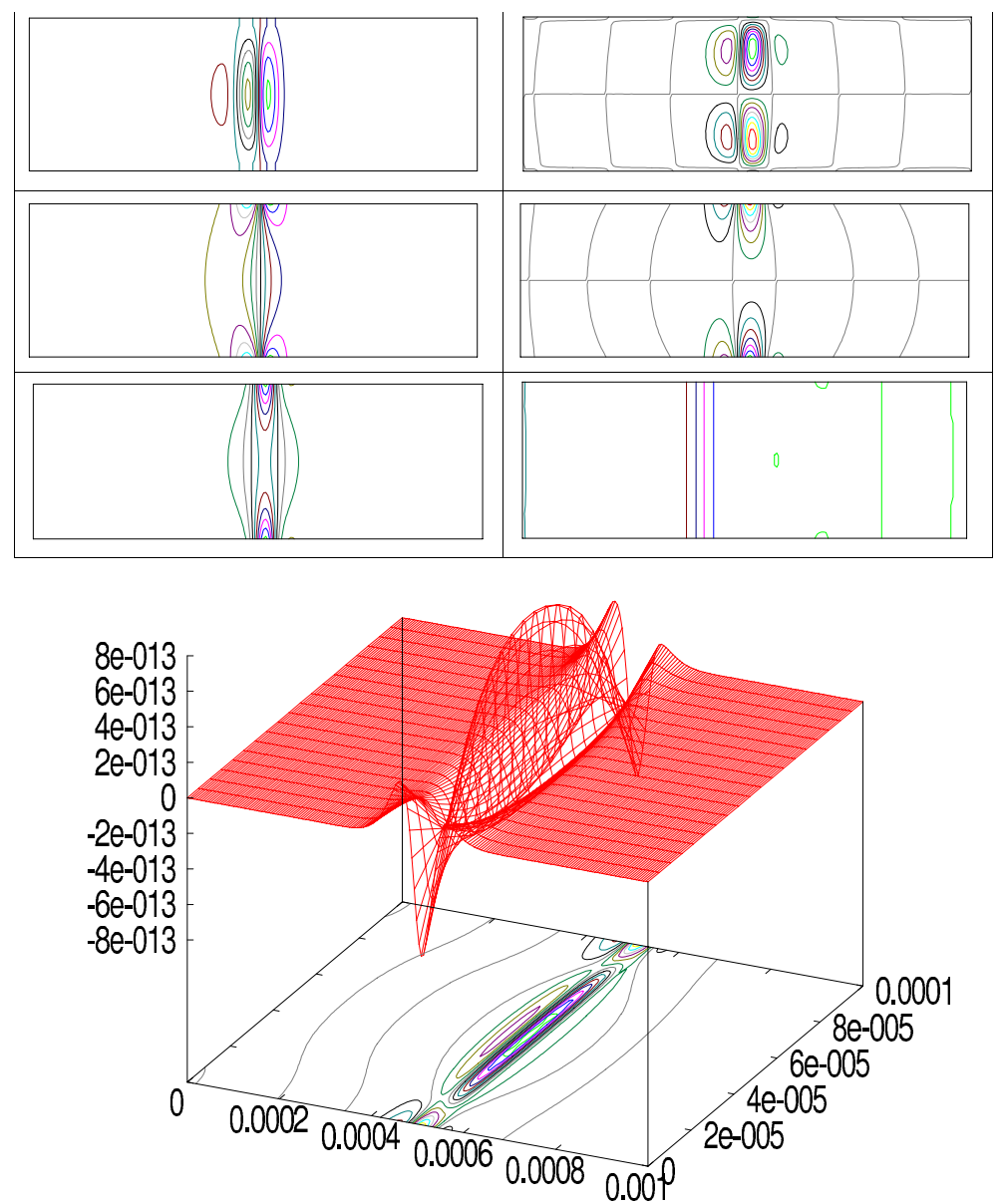

FiguRE 1. From top-left to bottom-right: instantaneous iso-contours for $\phi_{x}, \phi_{y}, u_{1}, u_{2}, p$ and $c^{3}$. Bottom figure shows iso-contours and surface of $\rho_{e}$ after $0.001 \mathrm{~s}$.

We chose the value $\alpha=4 \times 10^{4}$ in initial conditions (4.1) and $a=\left(d_{1}+d_{2}+d_{3}\right) / 3$ in (3.6). We tried also some other values for $a$, but the results were essentially the same as long as $a$ was of the same order of magnitude as diffusivity. This requirement is quite natural, considering that $a$ is the diffusivity for the auxiliary variable $\tilde{y}^{2}$.

As we showed above choosing the value of $\gamma$ in (4.1) approximately determines the ultimate stacking ratio. Otherwise choosing different values gave qualitatively similar results. The main difference is that reaching a higher stacking ratio takes longer time and hence a longer channel is needed.

Figure 1 shows instantaneous iso-contours of the electric field, velocity field, pressure, the stacked species $c^{3}$, as well as total charge density of sample species for $\gamma=50$. One can see that the propagating front for sample species where the stacking occurs is a little curved. Also it is clear that the errors in the total charge density are concentrated in this same region. To analyse the electroneutrality constraint and stacking in more detail we consider cross-section of the solution in the middle of the channel at different times.

Figure 2 shows the time histories for $c^{3}, \tilde{y}^{2}$ and $\rho^{e}$ when solving system (3.3) and (3.6) for $\gamma=50$ on around one second. Cross-sections are given at every $0.033 \mathrm{~s}$. One can clearly see that our method preserves better the electroneutrality constraint. With the original system one would have to remove one of the three species to guarantee electroneutrality. In addition, and perhaps even more importantly, our method respects the upper bound for stacking ratio given by $\gamma$ while the initial model overshoots this asymptotic value. 


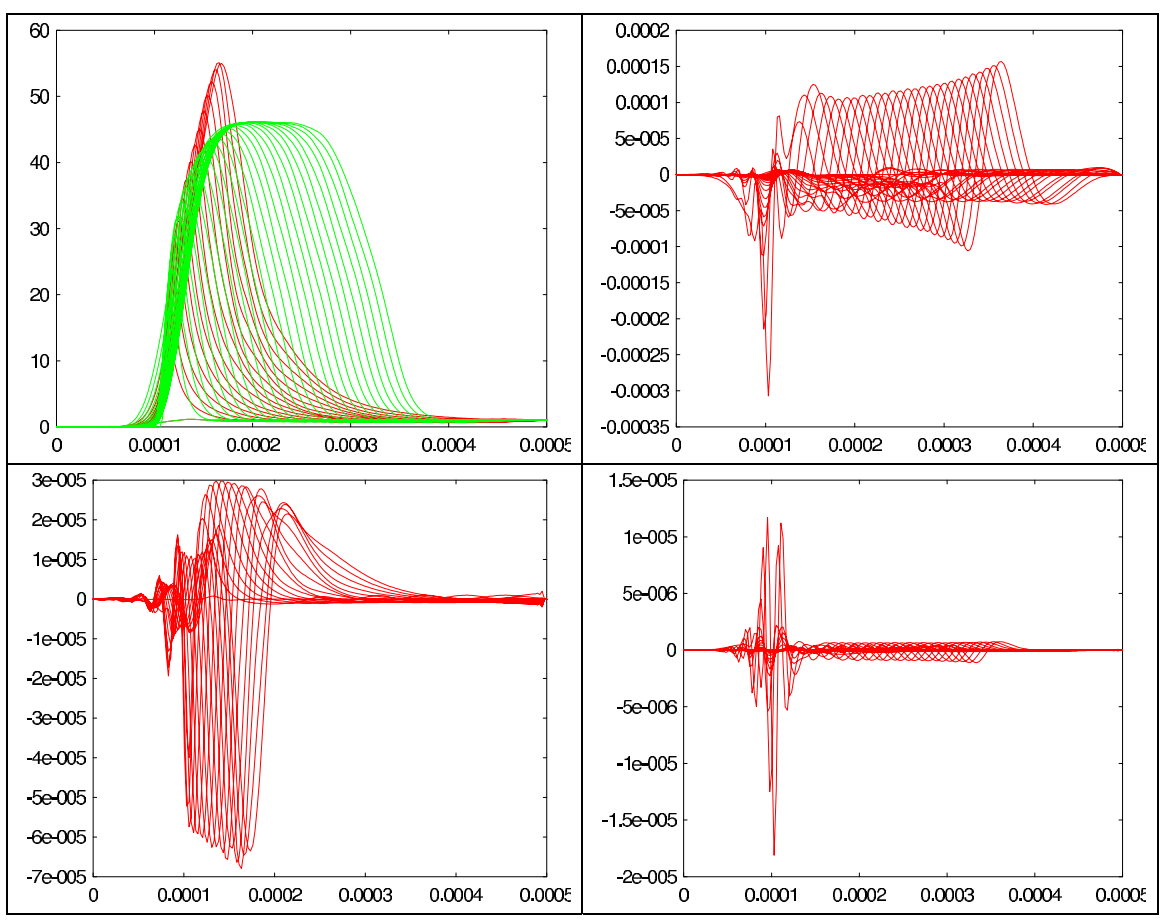

Figure 2. Evolution of $c^{3}$ (upper left) with the initial (3.3) and augmented (3.6) systems along the centerline of the channel for an applied electric field of $100 \mathrm{kV} / \mathrm{m}$ and $\gamma=50$. Upper right picture shows the evolution of $\tilde{y}^{2}$. Lower left (resp. right): $\rho^{e}$ with (3.3) (resp. with (3.6)).

We may interprete the results as follows. The solution is most sensitive to errors in the region where rapid changes occur, i.e. in the front where the sample species is stacked. Now the biggest errors are rather naturally in the same region, and because our method reduces significantly the electroneutrality error in this region, the concentrations are also much better resolved.

\section{Concluding REMarks}

We have shown above how our methodology, which was applied in $[12,13]$ to Stokes and Navier-Stokes problems, can also be used to improve existing solvers of microfluid systems. The results can be summarised as follows:

* The electroneutrality constraint has been satisfied without eliminating one of the species.

* The numerical solution with our approach satisfies the theoretical maximum stacking ratio.

* Computer implementation can be done by solving the equations cyclically and therefore the new equations of the augmented system have been easily introduced in an existing code.

In the present paper we did not discuss using the information contained in auxiliary variables so it seems the computation of these quantities did not serve for any useful purpose. However, since the auxiliary variables should be identically zero in exact solution these could be used as error indicators in adaptive numerical schemes. In [12] we indicated how this could be implemented.

There are also other physical models with algebraic constraints like the electroneutrality assumption. As an example we may cite the combustion problems where the conservation of mass leads to an algebraic constraint for mass fractions [16]. What presented here can therefore be applied to these systems as well. 
Acknowledgements. The authors would like to thank Professor Juan Santiago from Stanford Microfluidic Laboratory for his interest and comments on this manuscript.

\section{REFERENCES}

[1] M.S. Agranovich, Elliptic boundary problems, in Partial differential equations IX, M.S. Agranovich, Y.V. Egorov and M.A. Shubin Eds., Encyclopaedia of Mathematical Sciences 79, Springer (1997) 1-144.

[2] R. Brahadwaj, B. Mohammadi and J. Santiago, Design and optimization of on-chip capillary electrophoresis. Electrophoresis J. 23 (2002) 2729-2744.

[3] G. Bruin, Recent developments in electrokinetically driven analysis of microfabricated devices. Electrophoresis 21 (2000) 3931-3951.

[4] A. Douglis and L. Nirenberg, Interior estimates for elliptic systems of partial differential equations. Comm. Pure Appl. Math. 8 (1955) 503-538.

[5] P. Dudnikov and S. Samborski, Linear overdetermined systems of partial differential equations, in Partial Differential Equations VIII, M. Shubin Ed., Encyclopaedia of Mathematical Sciences 65, Springer (1996) 1-86.

[6] S.D. Eidelman, Parabolic equations, in Partial differential equations VI, M.A. Shubin Ed., Encyclopaedia of Mathematical Sciences 63, Springer (1994) 201-313.

[7] M.G. El Hak, The MEMS Handbook, Handbook series for Mechanical Engineering 7. CRC Press (2002).

[8] K. Krupchyk, W. Seiler and J. Tuomela, Overdetermined elliptic systems. Found. Comp. Math. 6 (2006) 309-351.

[9] K. Krupchyk and J. Tuomela, Completion of overdetermined parabolic PDEs. J. Symb. Comput. 43 (2008) 153-167.

[10] H. Lin, B. Storey, M. Oddy, C.-H. Chen and J. Santiago, Instability of electrokinetic microchannel flows with conductivity gradients. Phys. Fluids 16 (2004) 1876-1899.

[11] M. Marden, On the zeros of certain rational functions. Trans. Amer. Math. Soc. 32 (1930) 658-668.

[12] B. Mohammadi and J. Tuomela, Simplifying numerical solution of constrained PDE systems through involutive completion. ESAIM: M2AN 39 (2005) 909-929.

[13] B. Mohammadi and J. Tuomela, Involutive upgrades of Navier-Stokes solvers. Int. J. Comput. Fluid Dyn. 23 (2009) $439-447$.

[14] M. Oddy and J. Santiago, Multiple-species model for electrokinetic instability. Phys. Fluids 17 (2005) 1245-1278.

[15] B. Perthame, Transport equations in biology, Frontiers in Mathematics. Birkhäuser, Basel (2007).

[16] T. Poinsot and D. Veynante, Theoretical and Numerical Combustion. 2nd edn., R.T. Edwards, Inc. (2005).

[17] J.F. Pommaret, Systems of Partial Differential Equations and Lie Pseudogroups. Mathematics and its applications 14. Gordon and Breach Science Publishers (1978).

[18] R.F. Probstein, Physicochemical Hydrodynamics. Wiley (1995).

[19] W. Seiler, Involution - The Formal Theory of Differential Equations and its Applications in Computer Algebra. Algorithms and Computation in Mathematics 24. Springer, 2010.

[20] V.A. Solonnikov, On boundary value problems for linear parabolic systems of differential equations of general form. Trudy Mat. Inst. Steklov. 83 (1965) 3-163 (in Russian).

[21] D. Spencer, Overdetermined systems of linear partial differential equations. Bull. Amer. Math. Soc. 75 (1969) 179-239.

[22] T. Squires and S.R. Quake, Instability of electrokinetic microchannel flows with conductivity gradients. Rev. Mod. Phys. 77 (2005) 977-1026. 